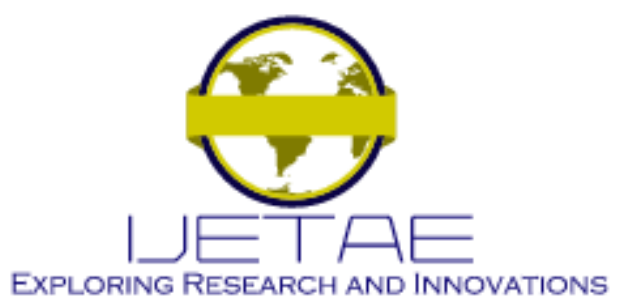

International Journal of Emerging Technology and Advanced Engineering

Website: www.ijetae.com (E-ISSN 2250-2459, Scopus Indexed, ISO 9001:2008 Certified Journal, Volume 12, Issue 02, February 2022)

\title{
Implementation of Dynamic and Efficient Virtual Channel Router for Network on Chip with Virtual Channel Arbitration Reduction and Parallel Switch Allocation Unit
}

\author{
Minakshi M. Wanjari ${ }^{1}$, Pankaj Agrawal ${ }^{2}$, Ravindra V. Kshirsagar ${ }^{3}$ \\ ${ }^{I}$ Electronics and Telecommunication Engineering Department, G. H. Raisoni College of Engineering, Nagpur, India \\ ${ }^{2}$ Electronics and Communication Engineering Department, Vidarbha Institute of Technology, Nagpur, India \\ ${ }^{3}$ Electronics Engineering Department, GGITS, Jabalpur, India.
}

\begin{abstract}
Network on chip (NoC) is a new method of interconnecting modules in system on chip (SoC). Now a day, it is believed that this new communication subsystem will replace the bus-based architecture and will become dominant in SoC design technology. The performance of NoC mainly depends on the router so it is considered as the crucial part in the NoC design. This paper describes the different factors which affect the NoC performance and provides solution to solve these problems. The proposed router has three architectural modifications which makes it dynamic and efficient as compare to generic virtual channel router. This paper introduced the dynamic buffer allocation scheme in which virtual channels and buffer slots are selected and allocated dynamically depending on the network traffic conditions in real time which in turn increases the throughput. In this paper, we have focused on optimizing the virtual channel arbitration (VA) unit design and switch allocation (SA) unit design as well as focusing the maximum utilization of buffer so that it will result in minimizing the latency, area and power. The source code of the router is written in VHDL hardware description language and the simulation and synthesizing the router is done in Xilinx ISE Design Suite 13.1. The parameters like area, frequency, delay, power, latency and throughput are calculated. The results of this modified virtual channel router are compared with the generic virtual channel router.
\end{abstract}

Keywords - Intellectual Property (IP), Network on Chip (NoC), Network Interface (NI), Switch allocation (SA), System-on-Chip (SoC), Virtual channel (VC), Virtual channel arbitration (VA).

\section{INTRODUCTION}

As the integration density is increasing and the feature size is decreasing, the interconnection between the components of SoC becomes a major factor on which the overall quality of a SoC depend.
Processing units in SoC are large in number so there is a requirement of interconnection system which can support large number of processing units but the system bus based SoC cannot meet these requirements due to the scalability issue. Long wires used in $\mathrm{SoC}$ also have number of issues like congestion in routing, noise coupling, and blockage. So, the overall solution to these problems is NoC architectures which uses a packet based communication $[1,2,3]$.

Router is the fundamental component of NoC which guides and coordinates the data flow in the network. It is said that the NoC performance is determined by the router architecture and is considered as the most important factor. The virtual channel router is also widely accepted option for Network-on-Chip [4].

A generic or conventional virtual channel router uses a static buffer allocation technique which results in blocking problem during the congestion called as Head-of-Line (HoL) blocking. The concept of buffer customization is introduced in [5] which decreasing the queue blocking probability which in turn improves the performance of the network.

Buffer implementation using registers takes a large area compared to other combinational logics and they consume much leakage [2]. Almost a 64 percent of router leakage power is consumed by buffers [6]. Therefore, the buffer design is considered as the important factor for the implementation of efficient NoC.

The malicious grant signals which occurred double, the effect of non-occurring grant signals and the effect of violating priority of signal occurring in both the arbitration logic units are critically important, as they result in flit losses and packet losses as well as degrading the performance [7]. 


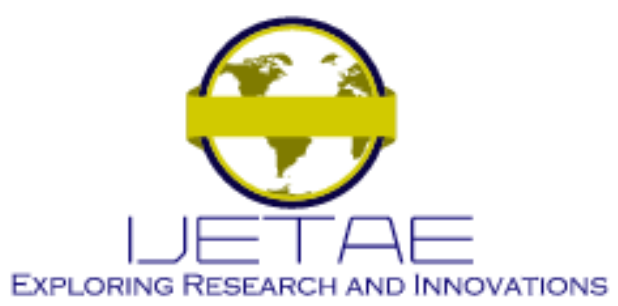

International Journal of Emerging Technology and Advanced Engineering

Website: www.ijetae.com (E-ISSN 2250-2459, Scopus Indexed, ISO 9001:2008 Certified Journal, Volume 12, Issue 02, February 2022)

So, here the objective was to modify the architecture of generic router by making three architectural modifications which could make it efficient as well as dynamic as compared to generic router.

The remaining paper is arranged in following way. Section II contain the literature review III presents NoC architecture; section IV describes about the generic/conventional NoC router architecture, section $\mathrm{V}$ describes the proposed router i.e., dynamic and efficient VC router, section VI is implementation and result and comparison of generic and modified router and the last section is conclusion.

\section{LITERATURE REVIEW}

In NoC architecture, a router transmits packet from a source router to a destination router through different intermediate nodes. If the head of the packet is blocked at the time data transmission then the router cannot transfer the packet. To remove this blocking problem, researchers proposed wormhole routing method. In wormhole routing method, the packet splits into number of flits. Now these flits can be transferred in a single transmission. To avoid the deadlock problem virtual channels are used and this also increases the throughput.

Various researchers have proposed different methods to solve various issues related to the $\mathrm{NoC}$ router architecture. Author Kim, J. introduced a low-cost, on-chip network router micro architecture by partitioning the crossbar, prioritizing packets in flight to simplify arbitration, and reducing the amount of buffers [8]. M.H. Ghadiry concluded a linear relation between number of virtual channels and energy dissipation. It is found that underutilized VCs result in energy dissipation. and an increase the number of VCs improve performance, but also increases latency[9]. Avinash Kodi, Ahmed Louri, Janet Wang combine two techniques of adaptive channel buffers and router pipeline bypassing to simultaneously reduce power consumption and improve performance [10]. Jimmy Kwa, Tor M. Aamodt presented an optimize design to reduce resource utilization. They proposed sharing Block RAMs between multiple router ports to store the high logic resource consuming virtual channel buffers and present BRS (Block RAM Split), a router architecture that implements the proposed optimization [11].
Jaya Suseela and Venkatesan Muthukum proposed router micro-architecture (LB-VC) consists of a feedback buffer structure and an asynchronous switching arbiter mechanism to achieve reduced area overhead and optimum buffer utilization, keeping inter-router latency and power consumption to a minimum [12]. Jian Wang, Yubai Li, and Qicong Peng proposed a novel buffer allocation algorithm that can be used to customize the router design in application-specific Network-on-Chip (NoC) and this method automatically assigns the buffer size for each input port in different routers across the NoC, such that the overall performance is maximized [13]. Authors Alireza Monemi, Chia Yee Ooi, and Muhammad Nadzir Marsono presented a two-clock-cycle latency NoC micro architecture. An efficient request masking technique is proposed to combine virtual channel (VC) allocation with switch allocation non-speculatively. Here proposed NoC architecture is optimized in terms of area overhead, operating frequency, and quality-of-service (QoS) [14]. Anuja Naik and Tirumale K. Ramesh, used a hybrid architecture by mixing buffered and bufferless routers using CLOS switch network. With this implementation they gain $32 \%$ reduction in area and $26 \%$ reduction in power compared to Crossbar switch of the same size [15]. Author Rashmiand Pavitha implemented a new buffer architecture which provide the efficient storage for multi data length packets, implemented a clock- gating circuit for improving power and added error termination logic for better functionality in address decoding. Ashok Kumar K and V. Karunakar Reddy discussed the issues on memory unit and proposed advanced FIFO based memory structure to enhance the speed data transfer for Bi directional NoC with a self configurable intercommunication channel [17].

\section{NOC ARCHITECTURE}

A NoC design consists of several intellectual property (IP) Cores of same type or different types linked in mesh pattern, shown in the Figure1. These IP Cores can be CPU, audio processing units, video processing units, memory units and wireless transceivers. Each IP Core is attached to a router which is a local to that IP c6ore through NI, i.e., network Interface. Each locally connected router is further attached to its neighbouring routers adjacent to it and forms a network called as on-chip network. 


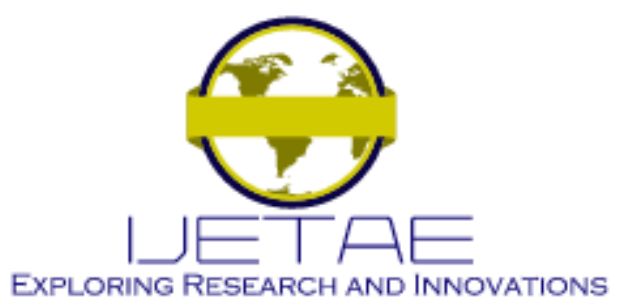

International Journal of Emerging Technology and Advanced Engineering Website: www.ijetae.com (E-ISSN 2250-2459, Scopus Indexed, ISO 9001:2008 Certified Journal, Volume 12, Issue 02, February 2022)

The network is a packet based network as the data transfer takes place in packets. The Network Interface module is responsible for packetizing and de-packetizing the data which are coming in and going out through the interconnected network.

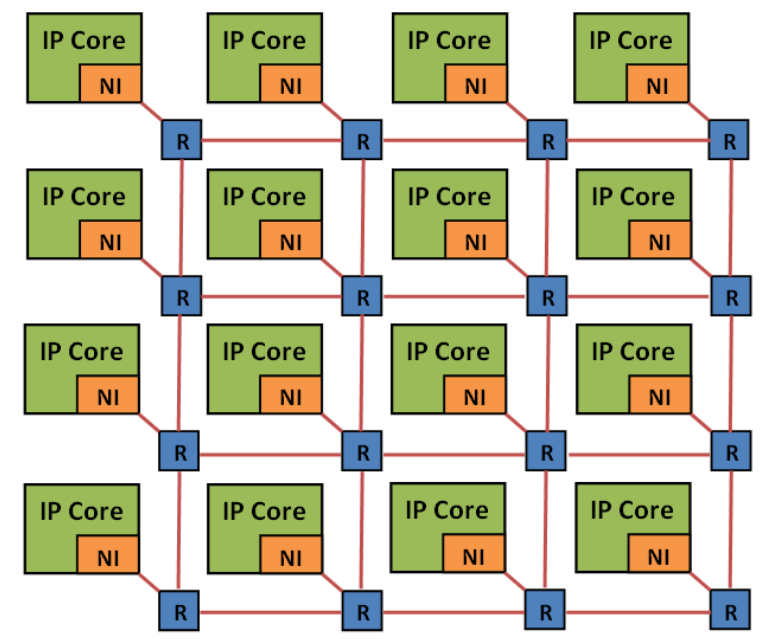

Figure 1. Generic NoC architecture

The IP Core with the NI module is called as a network node. Communication between the nodes takes place by transferring data packets to each other. By using different routing algorithms and flow control techniques, these data packets move forward into the network and reach to their destination.

The network topology is defined as the arrangement of interconnection of nodes (IP cores) using links (channels) in a network. Network topology describes about various things like network shape, connection between the cores and communication of cores with each other. Some of the topologies are star, tree, mesh, torus, butterfly and polygon [18]. More preference is given to the mesh topology because of the properties of having layout efficiency and having good electrical properties. One more advantage of being considering this as the addressing of on-chip resources is simple in mesh topology [19]. In mesh topology network, the wires are connected in $\mathrm{x}$ columns and $y$ rows. The processing elements or IP cores are connected to the routers and these routers are connected to the intersection point of the two wires. The addresses of routers as well as IP cores are given by these 2 dimensional or $\mathrm{x}-\mathrm{y}$ coordinates in mesh.

\section{GENERIC NoC Router ARCHITECTURE}

Generic network on chip (NoC) router architecture consist of five input ports and five output ports. Out of these five ports, four ports are connected to the routers of four directions East, West, North, South and one port is connected to the local IP core module. Each NoC router is connected to a single IP Core for simplifying the router complexity as well as minimizing the traffic congestion. The input or output channels can be a unidirectional links or bidirectional links, or even it can be a serial links also (as shown in Figure 2).

In virtual channel-based router, each input port has a many virtual channels and here it is total $\mathrm{v}$ virtual channels. Each virtual channel consists of some first in first out (FIFO) buffers, in this case, each FIFO buffer has dedicated $\mathrm{k}$ flits. The smallest unit in flow control is called as flit. Each network packet is a combination of different flits [20].

The NoC router consist of four different components (a) the $\mathrm{RC}$ unit which is for routing Computation, (b) the VA logic which is for Virtual Channel Arbitration, (c) the SA logic which is for Switch Allocation, and (d) the XBAR switch which is a Crossbar. The controlling logic is considered as the heart of $\mathrm{NoC}$ router.

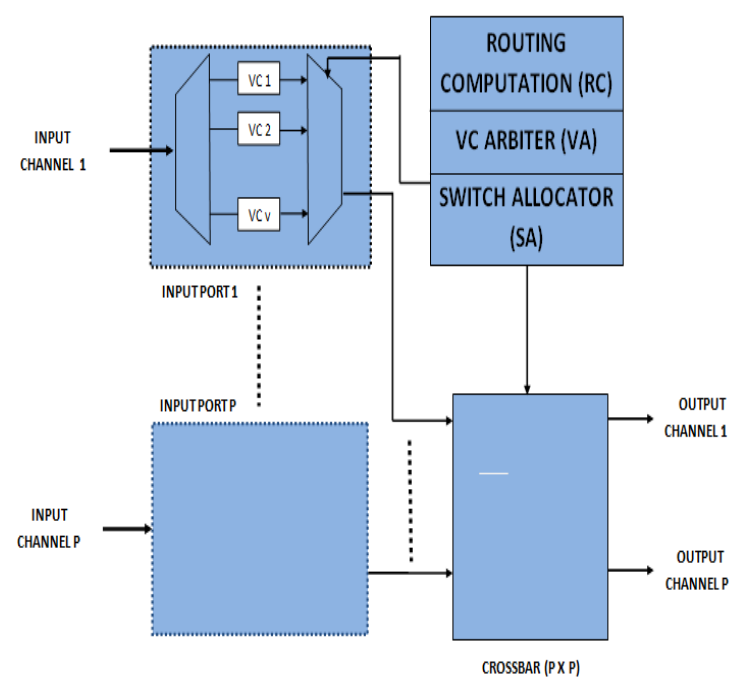

Figure 2. Generic NoC router architecture 


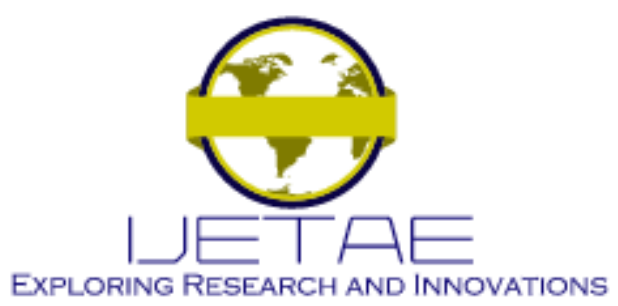

International Journal of Emerging Technology and Advanced Engineering

Website: www.ijetae.com (E-ISSN 2250-2459, Scopus Indexed, ISO 9001:2008 Certified Journal, Volume 12, Issue 02, February 2022)

\section{A. Routing Computation Unit}

The Routing Computation (RC) unit forwards the incoming packet towards the output channel. The incoming packet has header flit which consists of destination address. This destination address is used for routing of packets. The $\mathrm{RC}$ unit detects the valid output virtual channels (VC) for the appropriate Physical Channel. RC unit performs a "perpacket" operation.

\section{B. Virtual channel Arbitration Logic}

The Virtual channel arbitration (VA) unit performs the arbitration between all the packets which request access to the same Virtual Channel and decides the winning packet. This module includes two stages for arbitration because the routing technique does not indicate a required output $\mathrm{VC}$ channel for the requested output port. VA module is shown in Figure 3.

The first stage of virtual channel arbitration logic minimizes number of several incoming requesting signals from input virtual channel to one. VC arbitration logic consist of two arbitration stages. First stage consists of $\mathrm{Pv}$ number of v: 1 arbiters where one v: 1 arbiter is used for one input virtual channel. From each input virtual channel, several requests are there and they are arbitrated by first arbitration stage. The winning request from the first stage then proceeds towards the second stage of arbitration. The second arbitration stage consist of $\mathrm{Pv}$ number of $\mathrm{Pv}: 1$ arbiters. The one Pv: 1 arbiter is used for one output virtual channel. Just like routing computation logic, the arbitration in this module is also performed on "per-packet" and performed on header flits only [20].

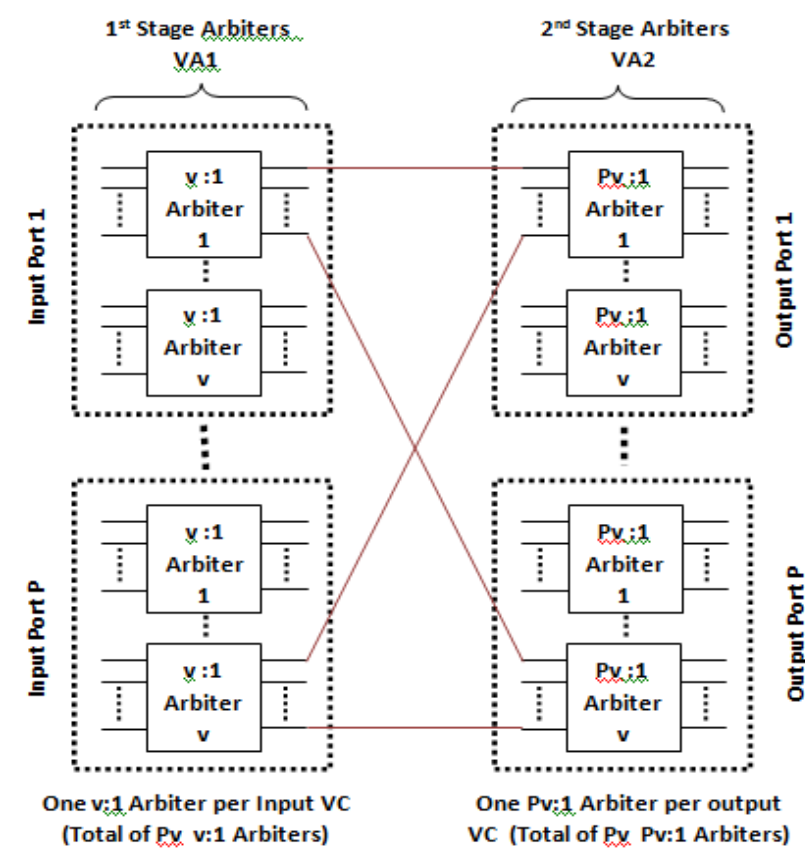

Figure 3. NoC router arbitration logic using VA unit.

\section{Switch Allocation}

The switch allocation (SA) unit arbitrates among all the virtual channels which request for accessing the crossbar and it allows only the winning request by giving permission. Switch allocation unit also includes two stages of arbitration. In first stage of switch allocation, a single port is shared by several virtual channels and in second stage arbitration of winning requests is performed for each output port. 


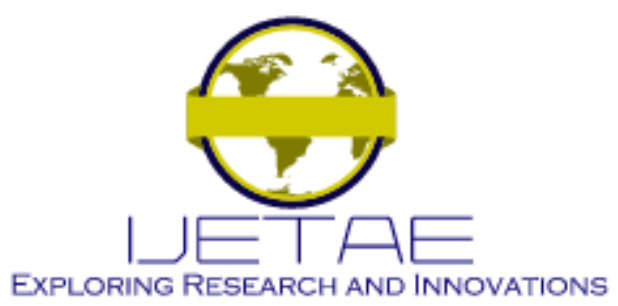

International Journal of Emerging Technology and Advanced Engineering

Website: www.ijetae.com (E-ISSN 2250-2459, Scopus Indexed, ISO 9001:2008 Certified Journal, Volume 12, Issue 02, February 2022)

These winning requests are from each input port. Crossbar control signals are set by the second stage of SA unit. The SA unit and VA unit are similar in structure. These arbiters are arranged in cascaded mode and are logically identical. But they differ in the count of arbiters included in module and the size as well. SA unit also consist of two stages. The first stage consists of P Number of v: 1 arbiters and second stage consist of $\mathrm{P}$ number of $\mathrm{P}: 1$ arbiters (shown in Figure 4). SA unit performs operation on "per-flit". The winning flits got access to the crossbar and then proceed towards the output port in the respective direction [20].

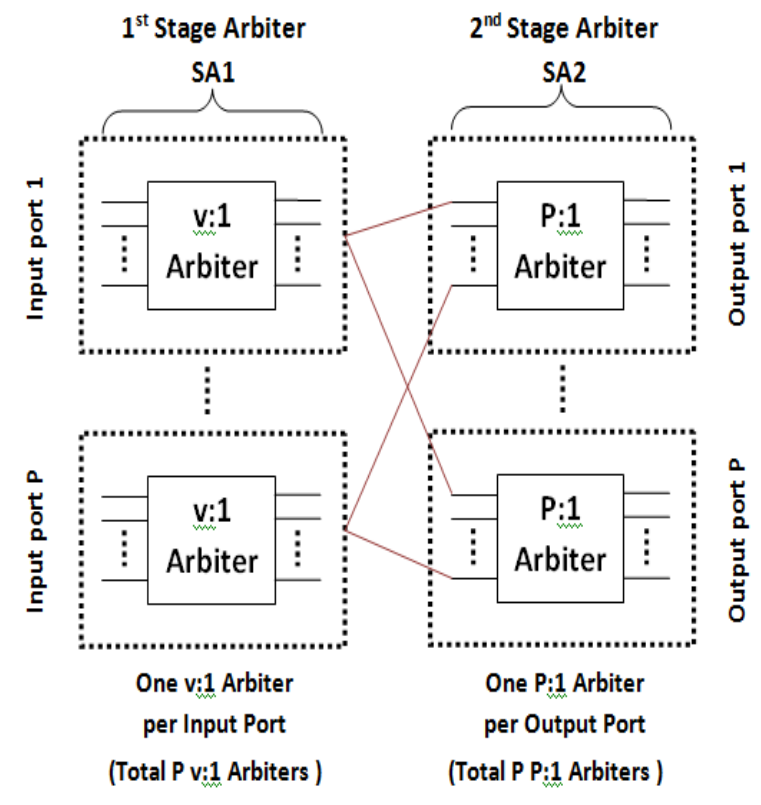

Figure 4. NoC router arbitration logic using SA unit.

\section{Crossbar}

As the flit receives a grant signal from the SA unit, it traverses through the crossbar switch and reach to its destination output port and this way it continues its journey throughout the network. As shown in the router architecture in Figure 2, single crossbar is shared by all the five input ports and number of flits has sent from different virtualchannel buffers of these input ports. Because of this sharing of crossbar by number of virtual channels, there is no need to increase crossbar size and it is not dependent on the number of virtual channels used [21].

\section{Dynamic AND EFFicient Virtual Channel Router}

In NoC architecture, packet starts its journey from source node, traverses through the different intermediate nodes with the help of a router and finally reaches to the destination node. But this data transmission suffers with a HoL blocking problem. The problem occurred during the data transmission, when a head packet gets blocked and cannot move forward in the network.

The remedial solution to the problem of head packet blocking is use of wormhole routing technique, in which a packet has split into number of flits and these flits are transmitted in a single transmission.

As the virtual channel buffers are implemented using registers and these buffers take large area and consume maximum leakage power [6]. It is said that total router leakage power consumed by buffers is about $64 \%$. Therefore, buffer design also plays an important role for implementation of efficient on-chip network [20].

The VA unit and SA unit are very important in router design as they result in flit or packet losses. These units are also responsible for degradation of performance as because of the malignant double occurring grants as well as the impact of getting no-grant signals and occurrence of violation of priority issue $[22,23]$. So, the aim was to do the three architectural modification in the existing generic router so that the router will become more dynamic and efficient which result in improving the performance of the router.

The following three modifications in architecture are

\section{A. A Dynamic Buffer Allocation Scheme}

In Dynamic buffer allocation scheme, virtual channels and buffer slots are selected dynamically in real time based on the traffic in network. This selection of buffer slots dynamically in run time results in increasing the buffer utilization and helps in decreasing the overall power consumption which in turn will improve the network performance. This dynamically allocated buffer structure (as shown in Figure 5) will minimize the HoL problem of head flit blocking. This scheme help in achieving higher throughput and reduces the chip area requirement as well as reduce the power consumption. 


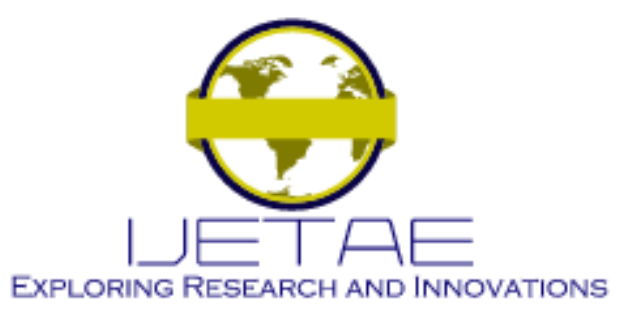

International Journal of Emerging Technology and Advanced Engineering Website: www.ijetae.com (E-ISSN 2250-2459, Scopus Indexed, ISO 9001:2008 Certified Journal, Volume 12, Issue 02, February 2022)

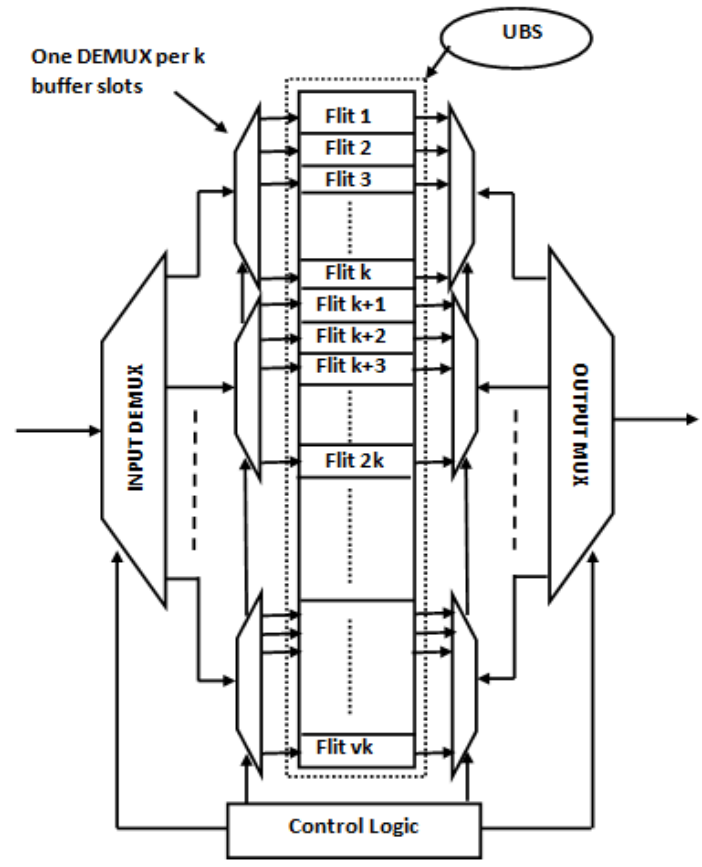

Figure 5. Dynamic buffer allocation scheme

\section{B. VA Reduction scheme}

A new virtual channel arbitration scheme with reduced arbitration stage is introduced in modified router. In this scheme (as shown in Figure 6) the first stage of arbitration is eliminated for synchronizing the appearance times of the inputs at the second stage of arbiters. In second stage the arbiter numbers are reduced from $\mathrm{Pv}$ to $\mathrm{P}$. While reducing the numbers of arbiters, the care has been taken that this reduction of arbiters should not affect the NoC performance.

The VA reduction module is efficient in avoiding packet losses which was afflicting generic virtual arbitration units. This module is area efficient as well as power efficient and helps in improving the performance of the router.

\section{Parallel SA Scheme}

In this new scheme of SA unit, the two stages of arbitration are arranged in parallel fashion as shown in Figure 7. This parallel arrangement of SA unit helps the switch allocation process by protecting from the appearance of double-grants as well as flit losses. The new parallel SA unit helps in improving the performance as this is considered to be the more efficient scheme as well as it improves the operating frequency by halving the SA critical path delay.

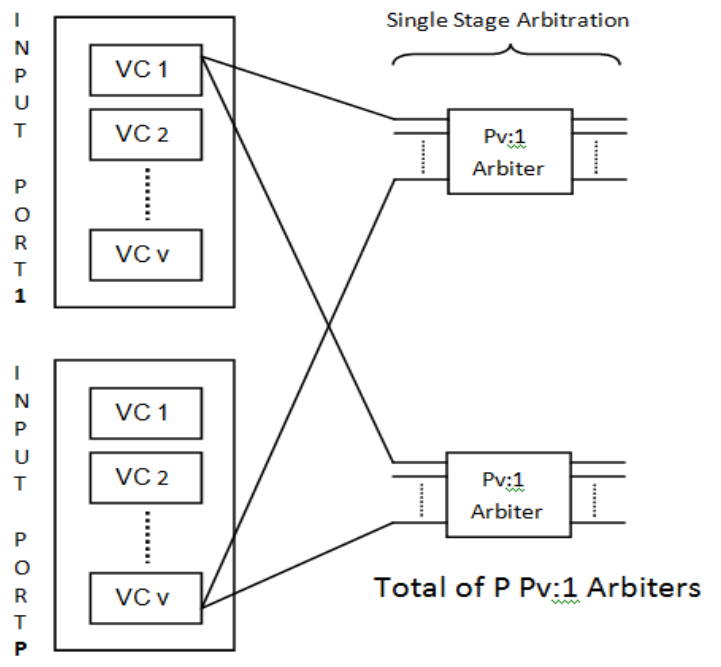

Figure 6. VA Reduction Unit.

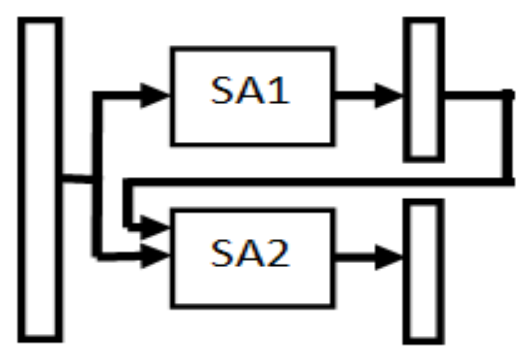

Figure 7. Parallel SA

\section{IMPLEMENTATION AND RESULT}

The router design code is implemented using VHDL (Hardware Description Language) on structural RTL (Register Transfer Level). The design synthesis and simulation is performed in Xilinx ISE Design Suite 13.1 software. This prototype is done in Spartan3 xc3s400. Simulation of the design verifies the design, its functionality and checks the performance of the design (as shown in figure 8).

In the process of simulation, numbers of stimulus are given and the corresponding responses are generated over time. The behaviour of the system is verified by test bench and tested for the routing pattern of different number of data packets. A test bench is a program whose purpose is to verify or check that the behaviour of system is as expected or not. Throughout the operation, the reset (rst) signal must be low. The input signals given to the router are data1, data2, data3, data4, data5. 


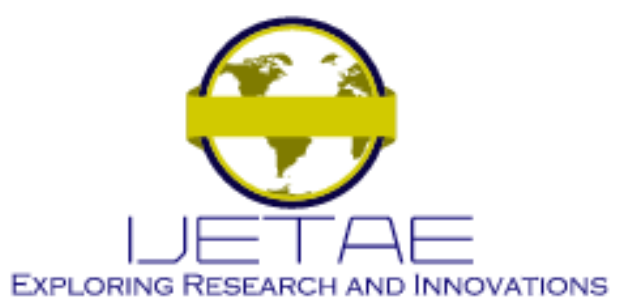

International Journal of Emerging Technology and Advanced Engineering

Website: www.ijetae.com (E-ISSN 2250-2459, Scopus Indexed, ISO 9001:2008 Certified Journal, Volume 12, Issue 02, February 2022)

The output signals are dout1, dout2, dout3, dout4, dout5 which shows the routing pattern. The first three bits of the input data are used as the select lines for de-multiplexer for directing the data from input channels towards the output channel. Table 1 shows the comparison between generic or conventional router and proposed modified router. Modified router requires less area (i.e., number of flip flops, number of slices and LUTS) as compare to generic router.
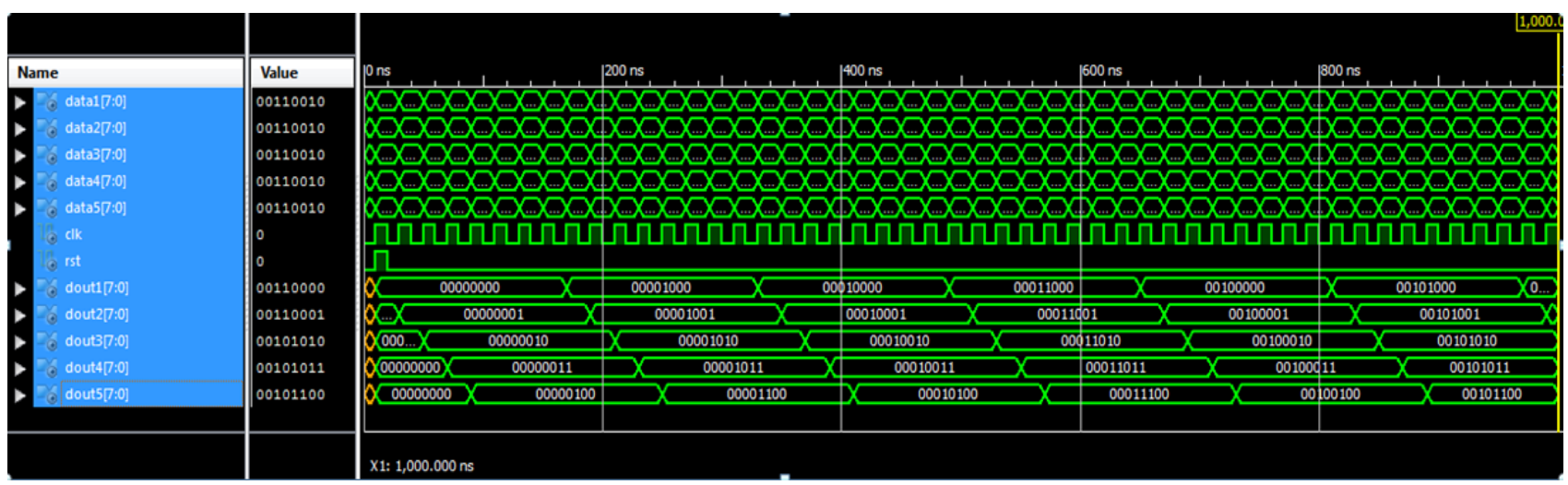

Figure 8. Proposed Router Simulation.

TABLE I

COMPARISON BETWEen GENERIC ROUTER AND Proposed MODIFIEd ROUTER.

\begin{tabular}{|l|l|l|}
\hline \multirow{2}{*}{ Parameters } & \multicolumn{2}{|c|}{ 5 port router } \\
\cline { 2 - 3 } & $\begin{array}{c}\text { Generic Router } \\
{[\mathbf{1 3}]}\end{array}$ & $\begin{array}{c}\text { Proposed Modified } \\
\text { Router }\end{array}$ \\
\hline Number of Slices & 2232 & 1395 \\
\hline Flip-Flops & 2962 & 1810 \\
\hline LUTS & 2164 & 1609 \\
\hline Frequency & $74.368 \mathrm{MHz}$ & $72.218 \mathrm{MHz}$ \\
\hline Clock period & $13.447 \mathrm{~ns}$ & $13.847 \mathrm{~ns}$ \\
\hline Average Latency & 39 clocks & 1.2 clocks \\
\hline Throughput \% & 20.008 & 41.2320 \\
\hline Power Consumption & $0.77 \mathrm{~W}$ & $0.79 \mathrm{~W}$ \\
\hline
\end{tabular}




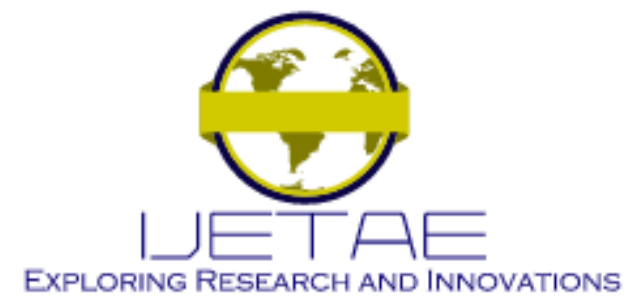

International Journal of Emerging Technology and Advanced Engineering

Website: www.ijetae.com (E-ISSN 2250-2459, Scopus Indexed, ISO 9001:2008 Certified Journal, Volume 12, Issue 02, February 2022)

\section{CONCLUSION}

As virtual-channel router is considered as one of the upcoming and assuring router architectures for Networkon-Chip which provides high throughput and less latency. But the disadvantage is that it has more buffer requirement, which is a resource-constrained for NoC. The parameters like VC buffer allocation scheme, VC and SA arbitration logic affect the network performance. To improve the performance of $\mathrm{NoC}$, three architectural modifications have described in this paper. First architectural modification is to use for allocating buffers dynamically in real time instead of statically using dynamic method for buffer allocation and it is based on real time traffic conditions of network. In second and third architectural modification, optimization of VA and SA units is done. These architectural modifications have done for maximizing the buffer utilization, power consumption, minimizing the latency and required area so as the throughput will increase and it can improve the performance of router. The comparison of modified virtual channel router with generic virtual channel router shows that, the modified router has less area, latency reduced comparably and throughput has increased almost double compared to generic router. But this makes the design complex, with increase in power consumption by $0.02 \mathrm{~W}$ and frequency reduced by $2.15 \mathrm{MHz}$. The three architectural modifications also help as a remedial solution for the occurrence of malignant grants, no-grant impact as well as violation of priority by identifying such possible consequences.

\section{REFERENCES}

[1] W. J. Dally, "Virtual-channel flow control," IEEE Trans. Parallel Distrib. Syst., vol. 3, no. 2, Mar. 1992, pp. 194-205.

[2] L. Benini and G. De Micheli, "Networks on chips: A new SoC paradigm," Computer, vol. 35, no. 1, 2002, pp. 70-78.

[3] W. J. Dally and B. Towles, "Route packets, not wires: On-chip interconnection networks," in DAC '01: Proceedings of the 38th Conference on Design Automation, Jun. 2001, pp. 684-689.

[4] P. Guerrier and A. Greiner, "A generic architecture for on-chip packet-switched interconnections," in DATE '00: Proceedings of the Conference on Design, Automation and Test in Europe, Mar. 2000, pp. 250-256.

[5] J. Hu, " U. Y. Ogras, and R. Marculescu, "System-level buffer allocation for application-specific networks-on-chip router design," IEEE Trans. on CAD of Integrated Circuits and Systems, vol. 25, no. 12, Jan. 2006, pp. 2919-2933.

[6] X. Chen and L. Peh, "Leakage power modeling and optimization in interconnection networks," in ISLPED '03: Proceedings of the 2003 International Symposium on Low Power Electronics and Design, Aug. 2003, pp. 90-95.

[7] Chrysostomos Nicopoulos, Vijaykrishnan Narayanan, Chita R. Das, "Network-on-Chip Architectures", Springer Science and Business Media LLC, 2010

[8] Kim, J., "Low-cost router microarchitecture for onchip networks". in 42nd Annual IEEE/ACM Int. Symp. Microarchitecture (MICRO42), NewYork, NY, USA, December 2009, pp. 255-266.
[9] M.H. Ghadiry, M.Nadi, M.T. Manzuri-Shalmani, D.Rahmati, "Performance and Power analysis of Routing Algorithms on NOC" 2008.

[10] Avinash Kodi, Ahmed Louri, Janet Wang, “ Design of EnergyEfficient Channel Buffers with Router Bypassing for Network-onChips (NoCs)", in Proceedings of the 10th Int'l Symposium on Quality Electronic Design, San Jose, CA , 16-18 March 2009, pp. $826-832$.

[11] Jimmy Kwa, Tor M. Aamodt, "Small Virtual Channel Routers on FPGAs Through Block RAM Sharing" in International Conference on Field-Programmable Technology 2012 ,Seoul National University, Seoul, Korea, 10-12 Dec., 2012

[12] Jaya Suseela and Venkatesan Muthukumar," Loopback Virtual Channel Router Architecture for Network on Chip",in Proceedings of the Ninth International Conference on Information TechnologyNew Generations. Apr. 2012, pp. 534 - 539.

[13] Jian Wang, Yubai Li, and Qicong Peng, "System-level Buffer Allocation for Application Specific Network-on-chip with Wormhole Routing”, (C) IETE Technical Review 2012

[14] Alireza Monemi, Chia Yee Ooi, andMuhammad NadzirMarsono, "Low Latency Network-on-Chip Router Microarchitecture using Request Masking Technique", (C) International Journal of Reconfigurable Computing 2015.

[15] Anuja Naik and Tirumale K. Ramesh , "Efficient Network on Chip (NoC) using heterogeneous circuit switched routers" , International Conference on VLSI Systems, Architectures, Technology and Applications (VLSI-SATA), 2016

[16] Rashmi A G and Pavitha U S, "Efficient Dynamic Router Architecture for Optimized Performance of NoC Systems", Proceedings of the International Conference on Inventive Research in Computing Applications (ICIRCA 2018) IEEE Xplore Compliant Part Number:CFP18N67-ART; ISBN:978-1-5386-2456-2

[17] Ashok Kumar K and V. Karunakar Reddy, "Advanced FIFO Structure for Router in Bi-NoC", Proceedings of the Fifth International Conference on Intelligent Computing and Control Systems (ICICCS 2021) IEEE Xplore Part Number: CFP21K74ART; ISBN: 978-0-7381-1327-2

[18] Swapna S., Swain, A.K., Mahapatra, K.K. Design and analysis of five port router for network on chip. Microelectronics and Electronics (PrimeAsia); 2012 Asia Pacific Conference on Postgraduate Research in, doi: 10.1109/Prime Asia. 2012. 6458626 ; 5-7 Dec. 2012; pp.51,55.

[19] Erika Cota, Alexandre de Morais Amory and Marcelo Soares Lubaszewski, "Reliability, Availability and Serviceability of Networks-on-Chip", Springer, 2012.

[20] C. A. Nicopoulos et al., "ViChaR: A dynamic virtual channel regulator for network-on-chip routers," in Proc. Int. Symp. Micro architecture, Dec. 2006, pp. 333-346.

[21] L. Peh and W. J. Dally, "A delay model and speculative architecture for pipelined routers," in Proc. Int. Symp. High-Performance Comput. Architecture, Jan. 2001, pp. 255-266.

[22] Chrysostomos Nicopoulos, Vijaykrishnan Narayanan, and Chita R. Das. "Network-on-Chip Architectures A Holistic Design Exploration”. DOI 10.1007/978-90-481-3031-3; (C) Springer Science+Business Media B.V.; 2009.

[23] É. Cota et al., "Reliability. Availability and Serviceability of Networks-on-Chip". DOI 10.1007/978-1-4614-0791-1_2; @ Springer Science+Business Media; LLC 2012.

[24] Minakshi M. Wanjari, Pankaj Agrawal, and Ravi V. Kshirsagar , "Implementation of Virtual Channel Router with VA and SA Arbitration Unit using VHDL", in International Journal of Engineering Applied Sciences and Technology (IJEAST), $2019 \mathrm{Vol}$ 4, Issue 5, September 2019, ISSN No. 2455-2143, Pages 158-163. 